\title{
Long-term outcomes of intradural cervical dorsal root rhizotomy for refractory occipital neuralgia
}

\author{
Abhiram V. Gande, MD, ${ }^{1}$ Srinivas Chivukula, MD, John J. Moossy, MD, ${ }^{1}$ William Rothfus, MD, ${ }^{3}$ \\ Vikas Agarwal, MD, ${ }^{3}$ Michael B. Horowitz, MD, ${ }^{2}$ and Paul A. Gardner, MD ${ }^{2}$ \\ ${ }^{1}$ University of Pittsburgh School of Medicine; Departments of ${ }^{2}$ Neurological Surgery and ${ }^{3}$ Radiology, University of Pittsburgh \\ Medical Center, Pittsburgh, Pennsylvania
}

\begin{abstract}
OBJECTIVE Occipital neuralgia (ON) causes chronic pain in the cutaneous distribution of the greater and lesser occipital nerves. The long-term efficacy of cervical dorsal root rhizotomy (CDR) in the management of ON has not been well described. The authors reviewed their 14 -year experience with CDR to assess pain relief and functional outcomes in patients with medically refractory $\mathrm{ON}$.
\end{abstract}

METHODS A retrospective chart review of 75 ON patients who underwent cervical dorsal root rhizotomy, from 1998 to 2012, was performed. Fifty-five patients were included because they met the International Headache Society's (IHS) diagnostic criteria for ON, responded to CT-guided nerve blocks at the C-2 dorsal nerve root, and had at least one follow-up visit. Telephone interviews were additionally used to obtain data on patient satisfaction.

RESULTS Forty-two patients (76\%) were female, and the average age at surgery was 46 years (range 16-80). Average follow up was 67 months (range 5-150). Etiologies of ON included the following: idiopathic (44\%), posttraumatic (27\%), postsurgical (22\%), post-cerebrovascular accident (4\%), postherpetic (2\%), and postviral (2\%). At last follow-up, 35 patients (64\%) reported full pain relief, $11(20 \%)$ partial relief, and $7(16 \%)$ no pain relief. The extent of pain relief after CDR was not significantly associated with ON etiology $(p=0.43)$. Of 37 patients whose satisfaction-related data were obtained, $25(68 \%)$ reported willingness to undergo repeat surgery for similar pain relief, while $11(30 \%)$ reported no such willingness; a single patient (2\%) did not answer this question. Twenty-one individuals (57\%) reported that their activity level/functional state improved after surgery, 5 (13\%) reported a decline, and $11(30 \%)$ reported no difference. The most common acute postoperative complications were infections in $9 \%(n=5)$ and CSF leaks in $5 \%(n=3)$; chronic complications included neck pain/stiffness in $16 \%(n=9)$ and upper-extremity symptoms in $5 \%(n=3)$ such as trapezius weakness, shoulder pain, and arm paresthesias.

CONCLUSIONS Cervical dorsal root rhizotomy provides an efficacious means for pain relief in patients with medically refractory $\mathrm{ON}$. In the appropriately selected patient, it may lead to optimal outcomes with a relatively low risk of complications.

http://thejns.org/doi/abs/10.3171/2015.6.JNS142772

KEY WORDS occipital neuralgia; outcomes; rhizotomy; dorsal; nerve root; pain

$\mathrm{O}$ CCIPITAL neuralgia $(\mathrm{ON})$ typically manifests as intense, paroxysmal, lancinating pain along the dermatomal distribution of the greater and/or lesser occipital nerve. Most cases of $\mathrm{ON}$ resolve with medical or physical therapies including nonsteroidal antiinflammatory medications, tricyclic antidepressants, neuroleptics, heat therapy, massage therapy, and local steroid injections. ${ }^{8,39}$ Pathological etiologies of ON include trauma, surgical injury, rheumatoid arthritis, nerve entrapment by hypertrophied C2 -3 facets, nerve compression by atlanto- epistrophic ligaments, Chiari malformations, spinal cord tumors, and idiopathic origins. ${ }^{8,17,30,33}$

When refractory, ON presents a treatment challenge. Although it has been recognized in the medical literature since $1821,{ }^{3}$ standardized care for refractory $\mathrm{ON}$ has been elusive. For the small subset of cases in which ON persists to inflict severe disabling pain, radiofrequency ablation (RFA) of the nerve, occipital neurectomy, neurostimulation, C-2 ganglionectomy, and partial or complete cervical dorsal root rhizotomy (CDR) are commonly used surgi-

ABBREVIATIONS CDR = cervical dorsal root rhizotomy; $C V A=$ cerebrovascular accident; $\mathrm{ON}=$ occipital neuralgia; $\mathrm{RFA}=$ radiofrequency ablation; $\mathrm{TN}=$ trigeminal neuralgia. 
cal treatments which provide varying degrees of pain relief. 16,32

The long-term efficacy of these procedures is unknown, making it difficult for patients and physicians to optimize refractory $\mathrm{ON}$ management. In this report, we describe our 14-year experience in a cohort of patients who underwent CDR for refractory ON. We prefer this surgical method in refractory ON for its potential blockade of all afferent sensory/pain transmission at the nerve root origin and its wide applicability regardless of etiology.

It is essential to have long-term follow-up for any intervention that addresses chronic pain syndromes. Multiple series on RF only have evaluated ON patients at a mean of 6- and 8-month end points, ${ }^{4,40}$ and other studies investigating neurectomy and dorsal root rhizotomy-intradural sectioning of dorsal nerve roots-have reported on small patient samples $(n<20)$ over median follow-up periods of less than 24 months. ${ }^{8,17,20}$ The present report on CDR provides a relatively long-term insight into a large population of patients receiving a standardized treatment.

\section{Methods \\ Data Collection}

Following institutional review board approval, we performed a retrospective chart review of all patients with ON treated by CDR at the University of Pittsburgh Medical Center (UPMC) between January 1998 and December 2012. After cross-referencing with UPMC's current procedural terminology (CPT) code database, we found 75 patients who were treated with complete intradural sectioning of upper cervical dorsal nerve roots $(\mathrm{C} 1-3)$ for medically refractory $\mathrm{ON}$.

Patients who did not meet the International Headache Society's diagnostic criteria for ON were excluded from the study; these criteria were namely the following: 1) paroxysmal stabbing pain, with or without persistent aching between paroxysms, in the distribution(s) of the greater, lesser, and/or third occipital nerve; 2) tenderness over the affected nerve; and 3) pain eased temporarily by local anesthetic block of the nerve..$^{14}$ Of 75 patients initially identified, 55 met the inclusion criteria of International Headache Society for ON, responded positively to CT-guided nerve blocks, and had at least one follow-up visit.

Of note, individual patient-reported descriptions of occipital pain and frequency of pain attacks had considerable variability. In addition, 4 patients (7\%) reported bilateral retro- or periorbital pain during a routine episode of $\mathrm{ON}$.

\section{Patient Population}

All patients were referred for neurosurgical consultation after medical therapy failing to treat ON. Medical records were reviewed for basic demographic information, etiologies, relevant medical comorbidities, surgical histories, ON symptomatic history, ON management history, intraoperative and immediate postoperative complications, follow-up neurological status, and current pain management. Gaps in the medical record were addressed during follow-up telephone calls. The demographic data are outlined in Table 1.

After excluding those without adequate follow-up (e.g.,
TABLE 1. Summary of presenting demographic features in 55 patients with $\mathrm{ON}$

\begin{tabular}{ll}
\hline \multicolumn{1}{c}{ Feature } & Value (\%) \\
\hline Females & $42(76)$ \\
\hline Age at op (yrs) & $46 \pm 15$ \\
\hline Mean \pm SD & $16-80$ \\
\hline Range & $55(100)$ \\
\hline Nerve block responsive & $15(27)$ \\
\hline History of AFP/TN & $13(24)$ \\
\hline History of migraines & $7(13)$ \\
\hline History of psychiatric disorders
\end{tabular}

AFP $=$ atypical facial pain

* Values are presented as the number (\%) of patients unless otherwise noted.

at least one record of a postoperative visit, letter correspondence, or telephone interview), our series contained 55 individuals. Forty-two patients $(76 \%)$ were female, and the average age at the time of surgery was 46 years (range $16-80$ years). Of note, 15 patients (27\%) had a history of atypical facial pain or trigeminal neuralgia (TN), 13 (24\%) had a history of migraine headaches, and 7 (13\%) had documented psychiatric disorders.

As seen in Table 2, idiopathic $(44 \%, \mathrm{n}=24)$, posttraumatic $(27 \%, \mathrm{n}=15)$, and postsurgical $(22 \%, \mathrm{n}=12)$ etiologies were the most common causes of refractory ON. In the 12 patients who had postsurgical ON, the etiology was presumed to be a prior retromastoid craniectomy for TN in 7 patients and "triple-root" dental surgery, multiple occipital shunt placements, $\mathrm{C} 1-2$ posterior fusion, suboccipital Chiari malformation decompression, and multiple anterior cervical discectomies and fusion in 1 patient each. In addition, 5 patients overall previously undergone RFA and a single patient had undergone peripheral neurectomy before opting for CDR. Two patients in our cohort reported onset of ON following a stroke, whereas another recalled ON-like symptoms after a bout with herpes zoster: One patient had a stroke in the cerebellum and the other patient sustained a microvascular hemispheric infarct that resulted in right-sided weakness. The exact mechanisms of $\mathrm{ON}$ in these patients is unclear, but the patients clearly related the onset of their symptoms to these events.

\section{CT Fluoroscopy-Guided Nerve Block}

CT fluoroscopy-guided nerve blocks of C1-3 were performed without anesthesia or sedation. ${ }^{17}$ Briefly, the necessary site of needle entry was marked with the help of an axial scout CT image from $\mathrm{C}-1$ to $\mathrm{C}-3$. Using ster-

TABLE 2. Etiologies of ON in 55 patients

\begin{tabular}{lc}
\hline Etiologies & No. of Patients (\%) \\
\hline Idiopathic & $24(44)$ \\
\hline Posttraumatic & $15(27)$ \\
\hline Postop & $12(22)$ \\
\hline Post-stroke & $2(4)$ \\
\hline Postherpetic & $1(2)$ \\
\hline Other postviral & $1(2)$ \\
\hline
\end{tabular}


ile technique, a 25-gauge $8.89-\mathrm{cm}$ straight spinal needle (Becton Dickinson) was carefully advanced to an exiting dorsal root under fluoroscopic guidance. Then, $0.5-2 \mathrm{ml}$ of $0.25 \%$ bupivacaine was injected to complete the block. The diagnosis of ON was confirmed if complete occipital pain relief and lack of trigger point stimulation was reported. A negative response was indicated by either incomplete pain relief or failure to prevent trigger point stimulation. All patients had nerve blocks administered by the same neuroradiologists (including coauthor W.R.).

\section{Surgical Technique}

All patients with positive responses to the dorsal root nerve blocks were deemed candidates for CDR. The distribution of dorsal roots sectioned is portrayed in Fig. 1. Only a small number of patients required bilateral C-2 rhizotomies based on their preoperative symptoms, but as a result the total number of $\mathrm{C}-2$ rootlets lesioned on each side is not equal to total cohort size $(\mathrm{n}=55)$. Occasionally, C-1 and/or partial C-3 or C-4 dorsal root rhizotomies were performed because of their visualized contributions to nerves arising from the $\mathrm{C}-2$ and $\mathrm{C}-3$ rootlets ${ }^{17}$ or due to subjective pain extension into the shoulder preoperatively. In the context of bilateral ON $(\mathrm{n}=13)$, clinical and surgical judgment dictated whether the bilateral rhizotomy would occur on the same day. In 11 cases, bilateral CDR was performed on the same day. In the remaining 2 cases, staged rhizotomies were performed 24 and 96 hours apart.

The surgical technique of CDR at our institution has been previously described by Kapoor et al. ${ }^{17}$ Briefly, under general anesthesia, patients are placed in the prone position their the head stabilized in a Mayfield head holder. A midline cervical incision is then made to eccentrically expose the ring of $\mathrm{C}-1$ and the lamina of $\mathrm{C}-2$ on the same side as the pain. Subsequently, the hemilamina of C-1, C-2 and part of C-3 are removed, which allows for opening of the dura to ultimately access the exiting nerve roots. The side-specific $\mathrm{C} 1-4$ dorsal nerve roots are then identified and sectioned, along with any unnamed intercalating branches among $\mathrm{C} 1-4$ that contributes to the $\mathrm{C}-2$ nerve root; care is taken to avoid any branches contributing to the spinal accessory nerve. To finish, the dura, fascia and incision are closed. Patients are typically discharged to home 2-3 days later. One patient underwent reoperation for incomplete sectioning of an aberrant C-2 dorsal root.

\section{Follow-Up}

Patient follow-up duration reflects the time from the date of surgery to that of the most recent postoperative clinic visit at the UPMC Department of Neurological Surgery, written correspondence, and/or a telephone interview at the time of the study. There was no predetermined protocol prior to this retrospective evaluation for regular evaluation of patients' symptoms. Therefore, all attempts were made to standardize follow-up over the phone if medical records were inadequate. Of note, the most recent follow-up for 37 patients was via telephone, whereas the most final follow-up for 18 patients was clinic visit or letter correspondence.

Pain relief was determined to be "full" if no further episodes of paroxysmal stabbing pain, constant headaches, or trigger point stimulation of such pain in the posterior scalp extending from the upper neck to the vertex vertically and to the ears laterally were absent; "partial" if some pain relief in preoperative ON symptoms was reported by the patients; and "none" if no discernible relief in headaches or pain was noted within the occipital nerve distribution.

Whenever possible, we also evaluated subjective parameters of patient satisfaction and functional status over the phone using the following questions: 1) "Considering the amount of pain relief you have gained from this procedure, was the surgery worthwhile or beneficial?" 2) "Considering the amount of pain relief you have gained from this procedure, would you get this surgery again if you could do it over?" 3) "Compared with how you were before the surgery, did your day-to-day activity level such as the ability to do chores, run errands, cook/bathe/dress/sleep or hold a job improve, worsen or stay about the same?"

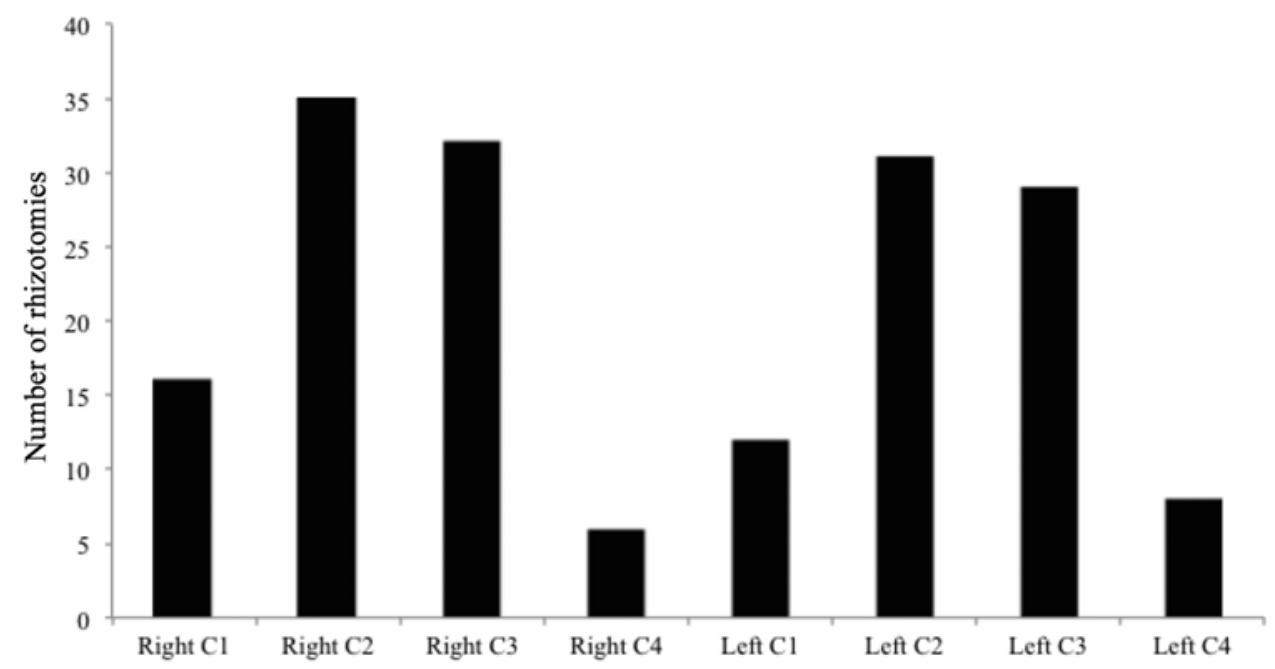

FIG. 1. Distribution of cervical roots that underwent intradural rhizotomy. Total number of rootlets lesioned is greater than the patient cohort size of 55 because several patients had bilateral ON $(n=13)$ and others had intraoperative evidence of contributions to their $\mathrm{C}-2$ rootlets from neighboring levels (C-1, C-3, and C-4). 
Although every attempt was made to reach all patients for telephone interviews, 18 patients had expired contact information, one patient died and one other declined to participate in the study.

\section{Statistical Analysis}

Basic descriptive statistics such as frequencies, means, and medians were calculated for all clinical variables. Cross-tabulations between etiologies and ultimate clinical outcomes were performed. Since all variables, except one (duration of follow-up), were categorical, Pearson chisquare (chi-square) statistics were used to evaluate associations of interest. All statistical tests were 2 sided, and $\mathrm{p}<0.05$ was considered statistically significant. Of note, 2 patients had staged dorsal rhizotomies for bilateral ON, making the total number of CDR procedures 57 in the 55 patients. However, for the purposes of this study, we treated the data from the staged procedures as a single, unified case for each patient.

\section{Results \\ Symptoms}

In the immediate postoperative period, all 55 patients developed numbness to touch in the occipital nerve distribution. At last documented follow-up (mean 67 months, median 52 months, range 5-150 months), 35 (64\%) of the patients achieved full pain relief from ON, $11(20 \%)$ patients obtained partial relief, and 7 (16\%) reported no improvement in their preoperative course of ON (Table 3). Among the 24 individuals who had no identifiable cause of ON, 17 (71\%) obtained full pain relief, 2 (8\%) had partial relief, and $5(21 \%)$ had no pain relief (Table 4).

Of the 15 patients who developed posttraumatic ON, 9 $(60 \%)$ had full resolution of ON symptoms, 5 (33\%) had partial relief, and $1(7 \%)$ had no relief. Of the 12 patients with a history of neck or skull base surgery, 7 (58\%) had full pain relief, $3(25 \%)$ had partial resolution, and $2(17 \%)$ had no occipital pain relief. Last, of the 13 patients with bilateral ON, 8 (62\%) reported full pain relief, 4 (31\%) had partial pain relief, and $1(7 \%)$ indicated no change in $\mathrm{ON}$.

Of the five patients with a history of RFA treatment for ON, $3(60 \%)$ achieved full pain resolution and $2(40 \%)$ reported partial pain relief after CDR. The only patient with a history of peripheral neurectomy indicated full pain relief following CDR.

Only 3 patients (5\%) had recurrent ON: it recurred approximately 12 months after CDR in 2 patients who relapsed with their full, baseline pain and approximately 2 months after CDR in the third patient, who still retained partial relief. The etiologies of ON in this subset of patients were posttraumatic $(n=2)$ and postsurgical $(n=1)$.

Treatment of ON prior to CDR did not influence the

TABLE 3. Pain relief outcomes following CDR in 55 patients

\begin{tabular}{cc}
\hline Pain Relief & No. of Patients $(\%)$ \\
\hline Full & $35(64)$ \\
\hline Partial & $11(20)$ \\
\hline None & $9(16)$ \\
\hline
\end{tabular}

TABLE 4. Pain relief outcomes stratified by etiology*

\begin{tabular}{lclll}
\hline & \multicolumn{3}{c}{ Postop Pain Relief } & \\
\cline { 2 - 4 } Etiology of ON & Full & Partial & None & Total \\
\hline Idiopathic & $17(71)$ & $2(8)$ & $5(21)$ & 24 \\
\hline Posttraumatic & $9(60)$ & $5(33)$ & $1(7)$ & 15 \\
\hline Postop & $7(58)$ & $3(25)$ & $2(17)$ & 12 \\
\hline Post-stroke & $1(50)$ & 0 & $1(50)$ & 2 \\
\hline Postherpetic & 0 & $1(100)$ & 0 & 1 \\
\hline Other postviral & $1(100)$ & 0 & 0 & 1 \\
\hline
\end{tabular}

* Values are presented the number (\%) of patients.

procedure's efficacy chi-square test $=1.7 ; \mathrm{p}=0.43$ ). The presence of psychiatric illness such as anxiety or major depression did not significantly affect pain outcomes (chi-square test $=0.1 ; p=0.99$ ). A history of migraines or comorbid pain disorders such as $\mathrm{TN}$, fibromyalgia, or arthritis did not significantly affect subjective pain relief; however, these patients did show a trend toward less relief (chi-square test $=4.7 ; \mathrm{p}=0.09$ ). The etiology of ON did not impact the efficacy of the procedure (chi-square test $=10.1 ; \mathrm{p}=0.43)$. Last, CDR provided similar results for those with bilateral versus unilateral ON (chi-square test $=1.8 ; \mathrm{p}=0.41)$.

\section{Patient Satisfaction}

To assess patient satisfaction, telephone interviews were conducted with 37 of the 55 patients. Of these, 25 (68\%) indicated that they would undergo this surgery again if they had to, $11(30 \%)$ that they would not do it again, and one (2\%) remained unsure (Table 5). The same proportions of patients stated that the surgery was worthwhile and/or beneficial, did not consider it worthwhile and/or beneficial or were unsure. Twenty-two patients (59\%) found that their activity level or functional state improved after surgery, 12 (32\%) found no change in their activity level, and $3(8 \%)$ stated that it had worsened. Full pain relief, but not partial relief, was significantly correlated with patient satisfaction (chi-square test $=13.4 ; \mathrm{p}=0.001$ ). Patients were more likely to report improved functional status after CDR (chisquare test $=16.3 ; \mathrm{p}=0.003$ ) if they obtained full pain relief compared with those who had unchanged pain status or partial levels of pain relief.

\section{Complications}

Acute complications occurred in 9 patients (16\%) and chronic complications in 12 (22\%) (Table 6). Acute complications included CSF leaks in 3 patients $(5.5 \%)$ and infections in 5 patients $(9.1 \%)$ (these were classified as wound infections, infections of the CNS, and urinary tract infections). In addition, a single patient complained of chest pain, which spontaneously resolved and was found to be noncardiac in origin. Chronic complications consisted of paresthesias and/or spasms in 8 patients (14.5\%), persistent pain in $5(9.1 \%)$, and torticollis $(1.8 \%)$, nerve (cranial nerve XI) apraxia (1.8\%), and nerve (cranial nerve XI) palsy (1.8\%) in 1 patient each. The latter complication occurred in a patient who presented with trapezius weak- 
TABLE 5. Telephone survey of patient satisfaction at last follow-up for 37 patients

\begin{tabular}{lccc}
\hline \multicolumn{1}{c}{ Question } & \multicolumn{3}{c}{ No. of Patients (\%) } \\
\cline { 2 - 4 } & Yes/lmproved & No/Worsened & Unsure/No Change \\
\hline Do you consider the surgery worthwhile and/or beneficial? & $25(68)$ & $11(30)$ & $1(2)$ \\
\hline Would you have this surgery again for the same pain relief? & $25(68)$ & $11(30)$ & $1(2)$ \\
\hline Did your functional activity improve? & $22(59)$ & $3(8)$ & $12(32)$ \\
\hline
\end{tabular}

ness and pain and 1 episode of paresthesia 3 months after CDR, subsequently recognized to be due to C3-4 subluxation and treated with posterior cervical decompression and fusion. Of note, chronic complications did not vary significantly between those with unilateral $(n=9)$ and bilateral $(n=3) \mathrm{ON}$ (chi-square test $=0.2 ; \mathrm{p}=0.9$ ).

\section{Discussion}

Occipital neuralgia is a rare and often incapacitating disorder that causes paroxysmal stabbing pain and chronic headache in the distribution of one or both occipital nerves. As with most neuropathic pain syndromes, conservative measures are initially employed for pain control before pursuing more invasive methods of management. This paper presents a large series of patients with prolonged follow-up after CDR for refractory ON.

\section{Etiologies}

Etiologies of ON can be better appreciated with knowledge of the unique anatomy in the upper cervical and suboccipital regions. The greater occipital nerve fibers arise mainly from the dorsal ramus of $\mathrm{C}-2$, with minor contributions from C-1 and C-3. The lesser occipital nerve arises

TABLE 6. Summary of complications following CDR in 55 patients

\begin{tabular}{cc}
\hline Complication & No. of Patients (\%) \\
\hline Acute complications & $3(5.5)$ \\
\hline CSF leak & $5(9.1)$ \\
\hline Infection & $3(5.5)$ \\
\hline Wound & $1(1.8)$ \\
\hline CNS & $1(1.8)$ \\
\hline UTI & \\
\hline Chronic complications & $8(14.5)$ \\
\hline Paresthesia/spasm/stiffness & $2(3.6)$ \\
\hline Occipital & $3(5.5)$ \\
\hline Neck & $3(5.5)$ \\
\hline Upper extremity & $8(14.5)$ \\
\hline Other neurological & $5(9.1)$ \\
\hline Persistent pain (neck) & $1(1.8)$ \\
\hline Torticollis (neck) & $1(1.8)$ \\
\hline Apraxia (arm) & $1(1.8)$ \\
\hline Nerve palsy (CN XI) & $1(1.8)$ \\
\hline Other & $1(1.8)$ \\
\hline Chest pain &
\end{tabular}

$\mathrm{CN}=$ cranial nerve; $\mathrm{UTI}=$ urinary tract infection. from the dorsal C-2 ramus to supply the upper/posterior auricular dermatome. ON patients invariably report symptoms in the distribution of the greater occipital, which is known to have six potential anatomical sites of compression. ${ }^{20,38}$ Additionally, anatomical studies have shown that the dorsal ramus of C-2 is unprotected by the atlantal pedicles and facets as it passes above the arch of the axis, which makes the nerve root vulnerable to compression injury. ${ }^{13}$ This becomes problematic during neck rotation, as it forces the atlantal facet to strike the axial arch, and neck extension, when the arches of the atlas and axis approximate one another. Other potential areas of injury along the greater occipital nerve's course include its path between the obliquus capitis inferior and semispinalis capitis muscles, its entry and exit points through the trapezius and semispinalis capitis muscles, and its piercing of the inferior nuchal line's tendinous insertions on the occipital bone..$^{20}$ Patients without a history of overt trauma or neck/ skull base surgery are deemed to have idiopathic causes of ON. Such patients may have accumulated subclinical insults to their occipital nerves, over years of repetitive neck extension, flexion, and rotatory movements.

In addition to idiopathic causes, postsurgical and traumatic insults are commonly cited etiologies (Fig. 2) of ON. ${ }^{1,8}$ Cases of postinfectious and postherpetic ON are also documented in the literature, ${ }^{5,15,19}$ but they are relatively uncommon. Two patients in our sample reported ON developing after a cerebral ischemic event. One patient had a cerebellar stroke and the other patient sustained a microvascular hemispheric CVA that resulted in rightsided weakness. The exact mechanism in these patients is unclear, and an extensive search of the literature did not yield any reports of post-CVA (stroke or TIA [transient ischemic attack]) ON, but there are cases of vascular anomalies, such as fenestrated vertebral arteries, that have led to thromboembolic events and occipital nerve compression. ${ }^{2,24,42}$ Direct compression that is coincidentally associated with these anomalies, changes in flow due to vessel thrombosis, or more complex alterations in pain pathways due to cortical or subcortical infarct involvement are all possibilities.

\section{Symptomatic Relief}

At an average follow-up of 67 months for our cohort, CDR provided full pain relief in $64 \%$, partial relief in $20 \%$, and no relief in $16 \%$ (Fig. 3). Hence, CDR provided some relief in at least $84 \%$ of our patients. These rates are consistent with those in previous reports from our institution. ${ }^{10,17}$ Of 17 patients in the study by Kapoor et al., $65 \%(\mathrm{n}=11)$ had full relief of symptoms, $12 \%(\mathrm{n}=2)$ had partial relief, and $24 \%(n=4)$ had no pain relief at a 


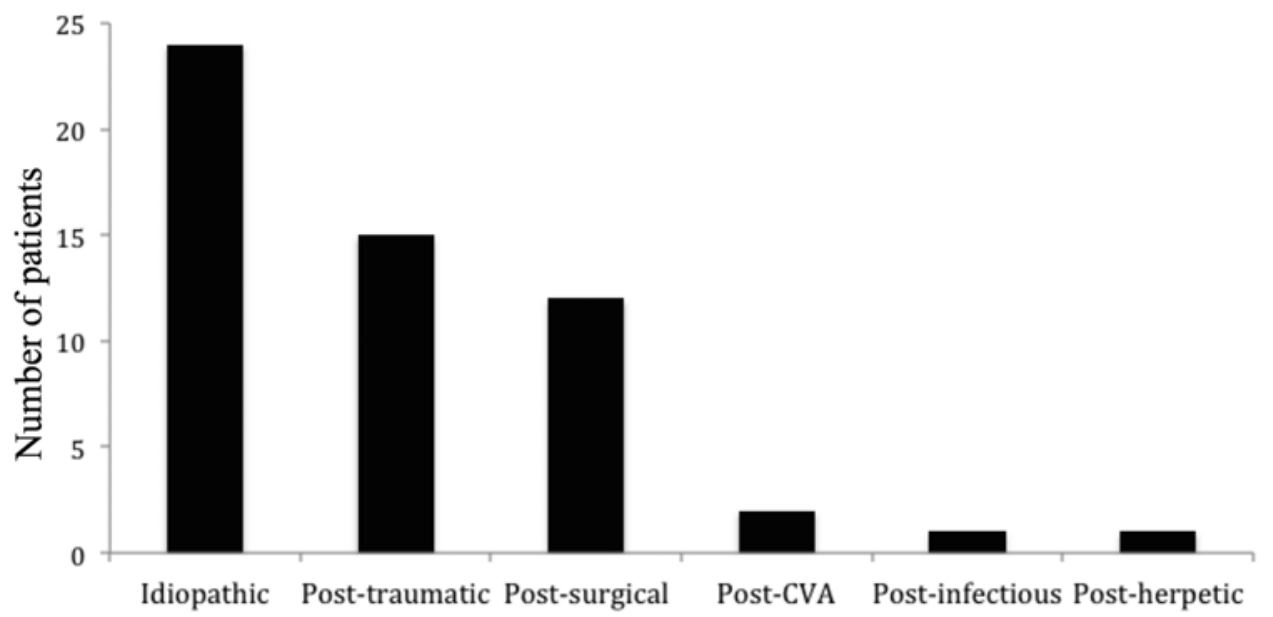

FIG. 2. Distribution of etiologies for ON in our cohort of 55 patients. The most common causes of ON in our sample were idiopathic, posttraumatic, and postsurgical. No significant relationship was found between these etiologies and the efficacy of CDR (chi-square test $=10.1 ; p=0.43$ ).

mean of 20 months after surgery. An older report, from 1972, on CDR treatment for ON found that 50\% ( $n=7)$ of the patients achieved "long-term" pain relief, whereas the remaining $50 \%$ had no changes in pain; the duration of follow-up is unknown. ${ }^{27}$ In 1954, Chambers evaluated 22 cases in which posterior root rhizotomy was performed for $\mathrm{ON}$; the follow-up period ranged from 3 to 78 months; ${ }^{3}$ $45 \%$ of their patients $(\mathrm{n}=10)$ achieved full pain resolution and $72 \%(n=16)$ endorsed $75 \%$ or more pain relief from the operation; $28 \%$ did not achieve favorable pain relief. Our study shows similar rates in a larger cohort and confirms the durability of the procedure.

\section{Alternatives to Dorsal Root Rhizotomy}

A close alternative to our technique is the partial root rhizotomy introduced by Sindou et al. in 1974 and implemented for ON by Dubuisson in the 1980s and 1990s. ${ }^{8,35}$ After conducting an extensive survey of spinal fibers at the dorsal root entry zone adjacent to the spinal cord, Sindou's team discovered that small-caliber fibers carrying nociceptive information are organized ventrolaterally at

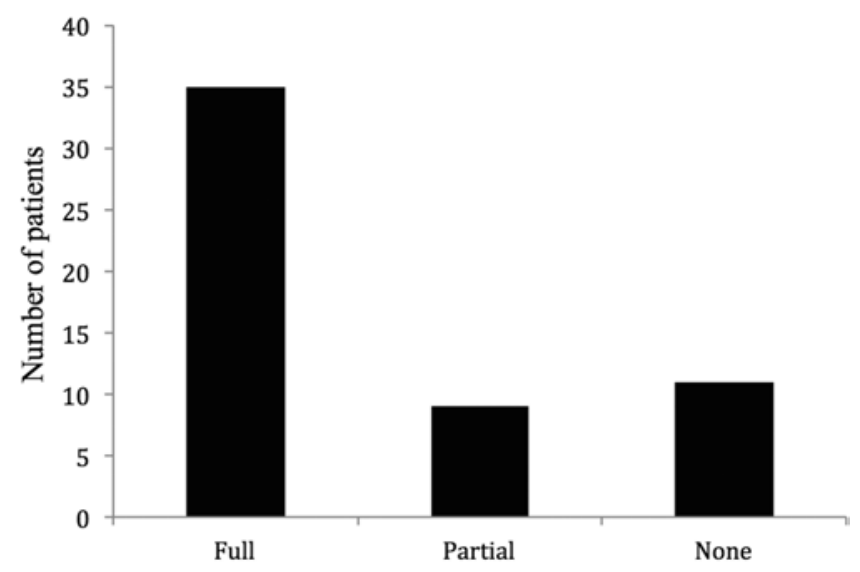

FIG. 3. Distribution of postoperative pain relief outcomes following intradural CDR in 55 patients. Only 3 patients $(5 \%)$ reported recurrence in ON pain after experiencing symptomatic relief postoperatively. the dorsal root entry zone. In contrast, larger-caliber fibers that serve cutaneous sensation and proprioception are organized dorsomedially. ${ }^{36}$ The most salient benefit of partial root rhizotomy over CDR is the preservation of cutaneous and proprioceptive sensation in the posterior scalp. Dubuisson and others have contended that complications such as vertigo or imbalance, secondary to the loss of afferent branches serving neighboring joints and muscle, can be avoided by selective sectioning of the ventrolateral fibers in the nerve root. ${ }^{8,35}$ In our series, no patient reported symptoms of ataxia or vertigo after the surgery (Table 6). Moreover, no patients sustained severe and chronic neuropathic pain in regions of numbness.

Interestingly, Dubuisson reported comparable pain relief outcomes in a mean follow-up period of 37 months after partial dorsal root rhizotomy: $71 \%$ of the procedures $(\mathrm{n}=10)$ yielded complete pain relief or partial pain relief responsive to nonprescription analgesics. ${ }^{8}$ The remaining 4 patients (29\%) continued with slightly better, but refractory, $\mathrm{ON}$ pain.

Several other alternatives to dorsal root rhizotomy exist and provide variable degrees of pain relief. C-2 ganglionectomy is commonly employed, and its primary benefits include removing the primary sensory neuronal cell bodies that mediate peripheral nociception, a relatively less invasive procedure to expose the ganglion compared with the intradural C-2 nerve root and a lower risk of CSF leaks. In a recent retrospective study by Pisapia et al., $66 \%$ of their patients $(n=19)$ had a good (partial relief) to excellent (full relief) outcome in a mean postprocedure followup duration of 5.6 years. ${ }^{29}$ However, only 18 patients in the study underwent C-2 ganglionectomy (the remaining 11 had received C-2 nerve decompression), and the study did not stratify outcomes between procedures. An older study by Lozano and colleagues explored outcomes in a cohort of 39 patients with a shorter follow-up (median 24 months) and found favorable results: $49 \%$ responding with excellent pain relief, $18 \%$ with partial pain relief, and $23 \%$ with poor outcomes. ${ }^{22}$ No cranial nerve morbidity or CSF leaks were noted in this study.

Percutaneous nerve blocks, using formulations of bu- 
pivacaine, lidocaine, or corticosteroids, are largely effective at providing immediate pain relief, even in refractory ON patients. ${ }^{16}$ However, nerve blocks provide only fleeting pain relief; one study noted that in $70 \%$ of its patients the pain recurred within 2 weeks and only $20 \%$ of the subjects had pain relief at 2.5 months after the block. ${ }^{21}$ Many patients endure through multiple nerve blocks before seeking better options such as RFA, decompression of nerve roots, and neurostimulation. A prospective study of RFA observed that only $53 \%$ of ON patients $(n=10)$ reported $50 \%$ or more pain relief at 6 months of follow-up. ${ }^{40} \mathrm{On}$ the other hand, a recent study in Korea followed patients for a mean of 7.5 months after RFA and found that $80 \%$ continued to have full pain relief, whereas $20 \%$ had partial pain relief. Significant pain reduction $(p<0.05)$ was also demonstrated using mean visual analog scale scores, which declined from 6.9 to 0.8 post-RFA. ${ }^{4}$ However, it remains to be seen whether RFA provides long-lasting pain relief, as most studies exploring this technique cite followup periods of fewer than 12 months. ${ }^{4,12}$

Imaging-positive impingement of nerve rootlets by C1-2 facet joint hypertrophy, fenestrated vertebral artery, ${ }^{24}$ or the atlantoepistrophic ligament ${ }^{30}$ may be amenable to surgical decompression. In the context of ON, successful decompressions by Poletti (C2-3 nerve roots) and Morikawa et al. (C1-2 nerve roots) underscore the utility of decompressive surgery in certain circumstances ${ }^{24,31}$ and the need to properly image patients to rule out these disorders. Peripheral nerve stimulation of the occipital nerve may also be a viable solution for refractory ON. Slavin et al. examined the use of a neurostimulator in 14 patients, in whom it provided some relief in 10 cases $(73 \%)$ and no pain relief in $4(29 \%) .{ }^{37}$ At a mean follow-up of 22 months, $70 \%$ of the 10 patients with stimulators continued to experience $50 \%$ or more reduction in pain; similar results are cited in other studies..$^{25,41}$ Despite the favorable outcomes associated with peripheral nerve stimulation, it is important to note that Slavin et al.'s and others' studies had relatively short follow-up durations. The permanent placement of a foreign device has significant disadvantages: limited battery life, risk of lead migration or scar formation leading to device failure, and infections that prompt device removal. ${ }^{23,37}$ These factors would undoubtedly affect longterm outcomes.

Lastly, peripheral neurectomies also provide some extent of pain resolution but pose postsurgical challenges. At 18 months of follow-up in one series, peripheral neurectomy provided $71 \%$ of patients $(n=15)$ with full pain relief, $19 \%(\mathrm{n}=4)$ with partial pain relief, and $10 \%(\mathrm{n}=2)$ with no symptomatic resolution. ${ }^{27}$ However, more than $50 \%$ of the patients reported partial return of posterior scalp sensation and $30 \%$ of patients had recurrence of pain, scalp hypersensitivity, dysesthesia, or painful neuroma formation. In contrast, only 3 patients (5\%) in our series had recurrent ON within a 12-month interval after CDR; 2 of them continued to have no pain relief and the other retained partial symptomatic relief.

We prefer intradural CDR in cases of refractory ON for its potential blockade of all afferent sensory/pain transmission at the nerve root origin, its durability, and its wide applicability regardless of etiology. The efficacy is borne out in this study in addition to durability, with only 3 patients (5\%) suffering ON recurrence.

\section{Patient Satisfaction}

We evaluated patient satisfaction after CDR among 37 individuals and found significant differences between those who attained full pain relief and those with partial/ no pain relief (see Results). Moreover, 68\% ( $\mathrm{n}=25)$ responded that they would have the surgery again for ON, regardless of the degree of pain relief they obtained (full or partial). Patients who did not experience any pain relief after CDR unanimously found no benefit and, thus, would not choose it again. With respect to functional improvement after the rhizotomy, 59\% of responders ( $\mathrm{n}=$ 22) found that their activity level improved considerably after the surgery; some of them were extremely grateful that they could sleep on a bed normally, get back to work, and reclaim their pre-ON status of general well-being. Thirty-two percent of patients $(n=12)$ found no difference in functional state, and $8 \%(\mathrm{n}=3)$ reported a decline in functional state due to chronic complications and/or inadequate ON pain relief (Table 6). Overall, our patients' satisfaction and self-reports of improvement in function are comparable to published in the literature ${ }^{3,17}$ and confirm the impact of successful treatment.

\section{Complications}

Although dorsal root rhizotomy is a viable and superior long-term solution for refractory ON, it is not exempt from postsurgical problems. Postoperative CSF leaks and infections occurred in 5\% (n=3) and 9\% $(\mathrm{n}=5)$ of the cases, respectively; all were promptly addressed with appropriate measures such as antibiotics and surgical interventions. On the other hand, chronic complications such as neck stiffness/pain/spasms, upper-extremity paresthesias and trapezius palsies, and occipital spasms/stiffness occurred in $16 \%(n=9), 5 \%(n=3)$, and $4 \%(n=2)$ of the patients, respectively. Patients often pursued physical therapy, massage therapy, and pharmacological options to manage their chronic complications. Persistent neck pain, stiffness, or spasms after spine surgery ${ }^{11}$ may reflect postlaminectomy kyphosis, ${ }^{18}$ muscular fatigue, ${ }^{7}$ and underlying comorbid illnesses (e.g., arthropathies).

There was one case of postoperative cranial nerve XI palsy in a patient who did not have it preoperatively and did not report any shoulder trauma after CDR. Given cranial nerve XI's origin in nerve rootlets arising from the upper cervical cord, care must be taken to identify these contributions, either visually and/or with nerve stimulation. Lastly, the C3-4 subluxation that occurred in a different patient is a known, but infrequent, complication of cervical laminectomies; care must be taken not to compromise the facet joints and increase the risk of postlaminectomy instability. ${ }^{6,34}$

Despite the chronic complication rate of $22 \%(n=12)$, most of the complications were relatively minor (neck stiffness) and many of our patients were grateful for the ON pain relief. No cases of deafferentation syndrome or anesthesia dolorosa, vertigo/imbalance, or death were observed in our series. All patients with lingering issues following their operation have sought further medical and 
physical treatments. Prevention of such complications may benefit from pre/postoperative muscle-strengthening exercises and postoperative cervical immobilization; this issue could be addressed in future prospective trials on CDR or posterior laminectomies.

Although many of our patients were on complicated medication regimens before surgery, cervical dorsal root rhizotomy provided remarkable pain relief for the majority of our patients. Chronic pain syndromes are often associated with mental illnesses, such as depression, posttraumatic stress disorder, and anxiety disorders. ${ }^{9,26,28}$ Despite significant comorbid medical and psychiatric diagnoses, summarized in Table 1, analysis of our patients with psychiatric comorbidities found no significant impact on the effectiveness of CDR ( $p=0.99)$. Nonetheless, extensive neuropsychological histories, social and occupational status, prior failed treatments, and unrealistic expectations need to be considered when selecting patients and determining outcomes after any pain-relieving intervention. Not surprisingly, coexisting pain disorders showed a trend toward decreased subjective pain relief but did not reach statistical significance (chi-square test $=4.7 ; \mathrm{p}=0.09$ ).

\section{Limitations}

Several limitations are present in our study. First, the lack of a control group with subjects who had a positive response to the CT-guided nerve block, but did not undergo rhizotomy, or a cohort that had undergone rhizotomy without a positive nerve block response, restricts the interpretation of our results. Additionally, we did not use validated tools to quantify pain, such as a visual analog scale or Likert scale, which can reduce the bias introduced by subjective interpretations of pain relief. Of 55 patients in the cohort, only 37 (67\%) were available for telephone follow-up to confirm pain relief and satisfaction outcomes. Although the mean and median lengths of follow-up in our study are significant, a larger prospective study with regular follow-up and comparison with other treatment modalities will allow us to draw stronger conclusions about the efficacy of CDR. Finally, there is a clear institutional bias toward using this approach over other alternatives that extends across providers.

\section{Conclusions}

Occipital neuralgia is a rare and debilitating pain syndrome that affects the cutaneous distribution of the greater and lesser occipital nerves. Regardless of etiology, CDR provided a clear majority of our patients with long-term pain relief and good functional outcomes. The small incidence of postoperative chronic morbidities, such as neck stiffness and persistent pain/spasms, may be addressed by better perioperative counseling and physical conditioning. Preoperative screening with occipital nerve block is strongly recommended.

\section{References}

1. Andrychowski J, Nauman P, Czernicki Z: [Occipital nerve neuralgia as postoperative complication. Views on etiology and treatment.] Neurol Neurochir Pol 32:871-876, 1998 (Polish)

2. Bernard TJ, Mull BR, Handler MH, Harned RK, Filley CM,
Kumpe DA, et al: An 18-year-old man with fenestrated vertebral arteries, recurrent stroke and successful angiographic coiling. J Neurol Sci 260:279-282, 2007

3. Chambers WR: Posterior rhizotomy of the second and third cervical nerves for occipital pain. J Am Med Assoc 155:431-432, 1954

4. Choi HJ, Oh IH, Choi SK, Lim YJ: Clinical outcomes of pulsed radiofrequency neuromodulation for the treatment of occipital neuralgia. J Korean Neurosurg Soc 51:281-285, 2012

5. Choi YK, Liu J: The use of 5\% lidocaine for prolonged analgesia in chronic pain patients: a new technique. Reg Anesth Pain Med 23:96-100, 1998

6. Clark CR (ed): The Cervical Spine. Philadelphia: Lippincott Williams \& Wilkins, 2005

7. Deutsch H, Haid RW, Rodts GE, Mummaneni PV: Postlaminectomy cervical deformity. Neurosurg Focus 15(3):E5, 2003

8. Dubuisson D: Treatment of occipital neuralgia by partial posterior rhizotomy at C1-3. J Neurosurg 82:581-586, 1995

9. Gureje O: Psychiatric aspects of pain. Curr Opin Psychiatry 20:42-46, 2007

10. Horowitz MB, Yonas H: Occipital neuralgia treated by intradural dorsal nerve root sectioning. Cephalalgia 13:354-360, 307, 1993

11. Hosono N, Yonenobu K, Ono K: Neck and shoulder pain after laminoplasty. A noticeable complication. Spine 21:19691973, 1996

12. Huang JH, Galvagno SM Jr, Hameed M, Wilkinson I, Erdek MA, Patel A, et al: Occipital nerve pulsed radiof requency treatment: a multi-center study evaluating predictors of outcome. Pain Med 13:489-497, 2012

13. Hunter CR, Mayfield FH: Role of the upper cervical roots in the production of pain in the head. Am J Surg 78:743-751, 1949

14. International Headache Society: The International Classification of Headache Disorders: 2nd edition. Cephalalgia 24:9-160, 2004

15. İpekdal Hİ, Yiğitoğlu PH, Eker A, Özmenoğlu M: Occipital neuralgia as an unusual manifestation of herpes zoster infection of the lesser occipital nerve: a case report. Acta Neurol Belg 113:201-202, 2012

16. Jürgens TP, Müller P, Seedorf H, Regelsberger J, May A: Occipital nerve block is effective in craniofacial neuralgias but not in idiopathic persistent facial pain. J Headache Pain 13:199-213, 2012

17. Kapoor V, Rothfus WE, Grahovac SZ, Amin Kassam SZ, Horowitz MB: Refractory occipital neuralgia: preoperative assessment with CT-guided nerve block prior to dorsal cervical rhizotomy. AJNR Am J Neuroradiol 24:2105-2110, 2003

18. Kaptain GJ, Simmons NE, Replogle RE, Pobereskin L: Incidence and outcome of kyphotic deformity following laminectomy for cervical spondylotic myelopathy. J Neurosurg 93 (2 Suppl):199-204, 2000

19. Kihara T, Shimohama S: Occipital neuralgia evoked by facial herpes zoster infection. Headache 46:1590-1591, 2006

20. Kim YS: The anatomy of the greater occipital nerve: part II. Compression point topography. Plast Reconstr Surg 128:322-323, 2011 (Letter)

21. Kuhn WF, Kuhn SC, Gilberstadt H: Occipital neuralgias: clinical recognition of a complicated headache. A case series and literature review. J Orofac Pain 11:158-165, 1997

22. Lozano AM, Vanderlinden G, Bachoo R, Rothbart P: Microsurgical C-2 ganglionectomy for chronic intractable occipital pain. J Neurosurg 89:359-365, 1998

23. McGreevy K, Hameed H, Erdek MA: Updated perspectives on occipital nerve stimulator lead migration: case report and literature review. Clin J Pain 28:814-818, 2012 
24. Morikawa K, Ohkawa N, Yamashita S: [Surgical decompression for the C-1 and C-2 sensory roots and upper cervical cord in a case with cervical myelopathy and occipital neuralgia due to bilateral fenestration of vertebral artery: a case report.] No Shinkei Geka 21:1035-1038, 1993 (Jpn)

25. Nörenberg E, Winkelmüller W: [The epifacial electric stimulation of the occipital nerve in cases of therapy-resistant neuralgia of the occipital nerve.] Schmerz 15:197-199, 2001 . (Ger)

26. Ohayon MM, Schatzberg AF: Using chronic pain to predict depressive morbidity in the general population. Arch Gen Psychiatry 60:39-47, 2003

27. Onofrio BM, Campa HK: Evaluation of rhizotomy. Review of 12 years' experience. J Neurosurg 36:751-755, 1972

28. Patten SB: Long-term medical conditions and major depression in a Canadian population study at waves 1 and 2 . J Affect Disord 63:35-41, 2001

29. Pisapia JM, Bhowmick DA, Farber RE, Zager EL: Salvage $\mathrm{C} 2$ ganglionectomy after $\mathrm{C} 2$ nerve root decompression provides similar pain relief as a single surgical procedure for intractable occipital neuralgia. World Neurosurg 77:362-369, 2012

30. Poletti CE: Proposed operation for occipital neuralgia: C-2 and $\mathrm{C}-3$ root decompression. Case report. Neurosurgery 12:221-224, 1983

31. Poletti CE, Sweet WH: Entrapment of the C2 root and ganglion by the atlanto-epistrophic ligament: clinical syndrome and surgical anatomy. Neurosurgery 27:288-291, 1990

32. Rasskazoff S, Kaufmann AM: Ventrolateral partial dorsal root entry zone rhizotomy for occipital neuralgia. Pain Res Manag 10:43-45, 2005

33. Scott M: Occipital neuralgia. Pa Med 71:85-88, 1968

34. Sim FH, Svien HJ, Bickel WH, Janes JM: Swan-neck deformity following extensive cervical laminectomy. A review of twenty-one cases. J Bone Joint Surg Am 56:564-580, 1974

35. Sindou M, Mifsud JJ, Boisson D, Goutelle A: Selective posterior rhizotomy in the dorsal root entry zone for treatment of hyperspasticity and pain in the hemiplegic upper limb. Neurosurgery 18:587-595, 1986

36. Sindou M, Quoex C, Baleydier C: Fiber organization at the posterior spinal cord-rootlet junction in man. J Comp Neurol 153:15-26, 1974
37. Slavin KV, Nersesyan H, Wess C: Peripheral neurostimulation for treatment of intractable occipital neuralgia. Neurosurgery 58:112-119, 2006

38. Standring S (ed): Gray's Anatomy, ed 40. London: Churchill Livingstone Elsevier, 2008, pp 435, 755

39. Vanelderen P, Lataster A, Levy R, Mekhail N, van Kleef M, Van Zundert J: 8. Occipital neuralgia. Pain Pract 10:137144,2010

40. Vanelderen P, Rouwette T, De Vooght P, Puylaert M, Heylen $\mathrm{R}$, Vissers K, et al: Pulsed radiofrequency for the treatment of occipital neuralgia: a prospective study with 6 months of follow-up. Reg Anesth Pain Med 35:148-151, 2010

41. Weiner RL, Reed KL: Peripheral neurostimulation for control of intractable occipital neuralgia. Neuromodulation 2:217-221, 1999

42. White JB, Atkinson PP, Cloft HJ, Atkinson JL: Vascular compression as a potential cause of occipital neuralgia: a case report. Cephalalgia 28:78-82, 2008

\section{Disclosures}

The authors report no conflict of interest concerning the materials or methods used in this study or the findings specified in this paper.

\section{Author Contributions}

Conception and design: Gardner, Gande. Acquisition of data: all authors. Analysis and interpretation of data: all authors. Drafting the article: Gardner, Gande, Chivukula, Moossy. Critically revising the article: all authors. Reviewed submitted version of manuscript: all authors. Approved the final version of the manuscript on behalf of all authors: Gardner. Statistical analysis: Gande, Moossy. Administrative/technical/material support: all authors. Study supervision: Gardner, Gande, Moossy, Rothfus, Agarwal.

\section{Correspondence}

Paul Gardner, Department of Neurological Surgery, University of Pittsburgh Medical Center, Ste. B-400, 200 Lothrop St., Pittsburgh, PA 15213.email: gardpa@upmc.edu. 